\title{
Solvability of a second-order Hamiltonian system with impulsive effects
}

Binxiang Dai* and Jia Guo

\section{*Correspondence:}

bxdai@csu.edu.cn

School of Mathematics and

Statistics, Central South University,

Changsha, Hunan 410075, China

\begin{abstract}
In this paper, a class of second-order Hamiltonian systems with impulsive effects are considered. By using critical point theory, we obtain some existence theorems of solutions for the nonlinear impulsive problem. We extend and improve some recent results.
\end{abstract}

MSC: 334B18; 34B37; 58E05

Keywords: Hamiltonian system; impulsive; critical point theory

\section{Introduction and main results}

Consider the second-order Hamiltonian systems with impulsive effects

$$
\left\{\begin{array}{l}
\ddot{u}(t)=\nabla F(t, u(t)), \quad \text { a.e. } t \in[0, T], \\
u(0)-u(T)=\dot{u}(0)-\dot{u}(T)=0, \\
\Delta \dot{u}^{i}\left(t_{j}\right)=I_{i j}\left(u^{i}\left(t_{j}\right)\right), \quad i=1,2, \ldots, N ; j=1,2, \ldots, p,
\end{array}\right.
$$

where $0=t_{0}<t_{1}<t_{2}<\cdots<t_{p}<t_{p+1}=T, u(t)=\left(u^{1}(t), u^{2}(t), \ldots, u^{N}(t)\right), I_{i j}: R \rightarrow R(i=$ $1,2, \ldots, N ; j=1,2, \ldots, p)$ are continuous and $F:[0, T] \times R^{n} \rightarrow R$ satisfies the following assumption:

(A) $F(t, x)$ is measurable in $t$ for every $x \in R^{n}$ and continuously differentiable in $x$ for a.e. $t \in[0, T]$ and there exist $a \in C\left(R^{+}, R^{+}\right), b \in L^{1}\left(0, T ; R^{+}\right)$such that

$$
|F(t, x)| \leq a(|x|) b(t), \quad|\nabla F(t, x)| \leq a(|x|) b(t)
$$

for all $x \in R^{n}$ and a.e. $t \in[0, T]$.

When $I_{i j} \equiv 0(i=1,2, \ldots, N ; j=1,2, \ldots, p),(1.1)$ is the Hamiltonian system

$$
\left\{\begin{array}{l}
\ddot{u}(t)=\nabla F(t, u(t)), \quad \text { a.e. } t \in[0, T], \\
u(0)-u(T)=\dot{u}(0)-\dot{u}(T)=0 .
\end{array}\right.
$$

In the past years, the existence of solutions for the second-order Hamiltonian systems (1.2) has been studied extensively via modern variational methods by many authors (see $[1-13])$.

(c) 2013 Dai and Guo; licensee Springer. This is an Open Access article distributed under the terms of the Creative Commons Attribution License (http://creativecommons.org/licenses/by/2.0), which permits unrestricted use, distribution, and reproduction in any medium, provided the original work is properly cited. 
When the gradient $\nabla F(t, x)$ is bounded, that is, there exists $g \in L^{1}\left(0, T ; R^{+}\right)$such that

$$
|\nabla F(t, x)| \leq g(t)
$$

for all $x \in R^{N}$ and a.e. $t \in[0, T]$, Mawhin-Willem in [1] proved the existence of solutions for problem (1.2) under the condition

$$
\lim _{|x| \rightarrow+\infty} \int_{0}^{T} F(t, x) d t=+\infty
$$

or

$$
\lim _{|x| \rightarrow+\infty} \int_{0}^{T} F(t, x) d t=-\infty .
$$

When the gradient $\nabla F(t, x)$ is bounded sublinearly, that is, there exist $f, g \in L^{1}\left(0, T ; R^{+}\right)$ and $\alpha \in[0,1)$ such that

$$
|\nabla F(t, x)| \leq f(t)|x|^{\alpha}+g(t)
$$

for all $x \in R^{N}$ and a.e. $t \in[0, T]$, Tang [2] proved the existence of solutions for problem (1.2) under the condition

$$
\lim _{|x| \rightarrow+\infty}|x|^{-2 \alpha} \int_{0}^{T} F(t, x) d t=+\infty
$$

or

$$
\lim _{|x| \rightarrow+\infty}|x|^{-2 \alpha} \int_{0}^{T} F(t, x) d t=-\infty
$$

which generalizes Mawhin-Willem's results.

For $I_{i j} \neq 0$, problem (1.1) gives less results (see [14-16]). In [14], Zhou and Li extended the results of [2] to impulsive problem (1.1); they proved the following theorems.

Theorem A [14] Assume that (A) and the following conditions are satisfied:

(h1) There exist $f, g \in L^{1}\left(0, T ; R^{+}\right)$and $\alpha \in[0,1)$ such that

$$
|\nabla F(t, x)| \leq f(t)|x|^{\alpha}+g(t)
$$

for all $x \in R^{n}$ and a.e. $t \in[0, T]$.

(h2) $\lim _{|x| \rightarrow+\infty}|x|^{-2 \alpha} \int_{0}^{T} F(t, x) d t=+\infty$.

(h3) For any $i=1,2, \ldots, N ; j=1,2, \ldots, p$,

$$
t I_{i j}(t) \geq 0, \quad \forall t \in R
$$

Then problem (1.1) has at least one weak solution.

Theorem B [14] Suppose that (A) and the condition (h1) of Theorem A hold. Assume that: (h4) $\lim _{|x| \rightarrow+\infty}|x|^{-2 \alpha} \int_{0}^{T} F(t, x) d t=-\infty$. 
(h5) For any $i=1,2, \ldots, N ; j=1,2, \ldots, p$,

$$
t I_{i j}(t) \leq 0, \quad \forall t \in R
$$

(h6) There exist $a_{i j}, b_{i j}>0$ and $\beta_{i j} \in(0,1)$ such that

$$
\left|I_{i j}(t)\right| \leq a_{i j}+b_{i j}|t|^{\alpha \beta_{i j}}, \quad \forall t \in R, i=1,2, \ldots, N ; j=1,2, \ldots, p .
$$

Then problem (1.1) has at least one weak solution.

Let

$$
F(t, x)=f(t)|x|^{1+\alpha}+C(x)-\frac{\lambda}{2}|x|^{2}+(h(t), x),
$$

where $C(x)$ is convex in $R^{N}$ (e.g., $\left.C(x)=\frac{\lambda}{2}\left(\left|x^{1}\right|^{4}+\left|x^{2}\right|^{2}+\cdots+\left|x^{N}\right|^{2}\right)\right), \lambda<\frac{4 \pi^{2}}{T^{2}}, f \in$ $L^{1}[0, T]$ satisfying $\int_{0}^{T} f(t) d t>0\left(e . g ., f(t)=\frac{2 T}{3}-t\right), 0<\alpha<1, h \in L^{1}\left(0, T ; R^{N}\right)$ and $x=$ $\left(x^{1}, x^{2}, \ldots, x^{N}\right) \in R^{N}$. It is easy to see that $F(t, x)$ satisfies the condition (h2) but does not satisfy the condition (h1). The above example shows that it is valuable to further improve Theorem A.

Let

$$
F(t, x)=f(t)|x|^{1+\alpha}+C(x)-\frac{\lambda}{2}|x|^{2}+(h(t), x),
$$

where $C(x)$ satisfies that the gradient $\nabla C(x)$ is Lipschitz continuous and monotone in $R^{N}$ (e.g., $\left.C(x)=\frac{\lambda}{2}\left(\left|x^{1}\right|+\left|x^{2}\right|^{2}+\cdots+\left|x^{N}\right|^{2}\right)\right), 0<\lambda<\frac{4 \pi^{2}}{T^{2}}, f \in L^{1}[0, T]$ satisfies $\int_{0}^{T} f(t) d t>0$ (e.g., $\left.f(t)=\frac{T}{3}-t\right), 0<\alpha<1, h \in L^{1}\left(0, T ; R^{N}\right)$ and $x=\left(x^{1}, x^{2}, \ldots, x^{N}\right) \in R^{N}$. It is easy to see that $F(t, x)$ satisfies the condition (h4) but does not satisfy the condition (h1). The above example shows that it is valuable to further improve Theorem $B$.

In this paper, we further study the existence of solutions for impulsive problem (1.1). Our main results are the following theorems.

Theorem 1.1 Suppose that $F(t, x)=H(t, x)+G(x)$ satisfies assumption (A) and the following conditions hold:

(H1) There exist $f, g \in L^{1}\left(0, T ; R^{+}\right)$and $\alpha \in[0,1)$ such that

$$
|\nabla H(t, x)| \leq f(t)|x|^{\alpha}+g(t)
$$

$$
\text { for all } x \in R^{N} \text { and a.e. } t \in[0, T] .
$$

(H2) There exists a positive number $\lambda<\frac{4 \pi^{2}}{T^{2}}$ such that

$$
(\nabla G(x)-\nabla G(y), x-y) \geq-\lambda|x-y|^{2}
$$

for all $x, y \in R^{n}$.

(H3)

$$
\lim _{|x| \rightarrow+\infty}|x|^{-2 \alpha} \int_{0}^{T} F(t, x) d t=+\infty .
$$


(H4) For any $i=1,2, \ldots, N ; j=1,2, \ldots, p$,

$$
t I_{i j}(t) \geq 0, \quad \forall t \in R
$$

Then impulsive problem (1.1) has at least one weak solution.

Remark 1.1 Theorem 1.1 generalizes Theorem A, which is a special case of our Theorem 1.1 corresponding to $G(x) \equiv 0$.

Example 1.1 Let $N=3, p=1$. Consider the following impulsive problem:

$$
\left\{\begin{array}{l}
\ddot{u}(t)=\nabla F(t, u(t)), \quad \text { a.e. } t \in[0, T], \\
u(0)-u(T)=\dot{u}(0)-\dot{u}(T)=0, \\
\Delta \dot{u}^{i}\left(t_{1}\right)=I_{i 1}\left(u^{i}\left(t_{1}\right)\right), \quad i=1,2,3,
\end{array}\right.
$$

where

$$
\begin{aligned}
& F(t, x)=\left(\frac{2 T}{3}-t\right)|x|^{\frac{3}{2}}-\lambda \cos x^{1}+(h(t), x), \\
& h \in L^{1}\left(0, T ; R^{3}\right), \quad I_{i 1}(t)=t^{\frac{1}{3}} \quad(i=1,2,3) .
\end{aligned}
$$

Take

$$
G(x)=-\lambda \cos x^{1}, \quad x=\left(x^{1}, x^{2}, x^{3}\right) \in R^{3},
$$

which is bounded and

$$
H(t, x)=\left(\frac{2 T}{3}-t\right)|x|^{\frac{3}{2}}+(h(t), x),
$$

$\alpha=\frac{1}{2}, f(t)=\frac{3}{2}\left(\frac{2 T}{3}-t\right), g(t)=|h(t)|$. Then all the conditions of Theorem 1.1 are satisfied. According to Theorem 1.1, the above problem has at least one weak solution. However, $F$ does not satisfy the condition (h1) in Theorem A. Therefore, our result improves and generalizes the Theorem A.

Theorem 1.2 Suppose that $F(t, x)=H(t, x)+G(x)$ satisfies assumption (A) and the condition (H1) of Theorem 1.1. Furthermore, assume that

(H5) There exist $0 \leq A<\frac{4 \pi^{2}}{T^{2}}, B \geq 0$ such that

$$
|\nabla G(x)-\nabla G(y)| \leq A|x-y|+B
$$

for all $x \in R^{n}$.

(H6)

$$
\lim _{|x| \rightarrow+\infty}|x|^{-2 \alpha} \int_{0}^{T} F(t, x) d t=-\infty
$$


(H7) For any $i=1,2, \ldots, N ; j=1,2, \ldots, p$,

$$
t I_{i j}(t) \leq 0, \quad \forall t \in R
$$

(H8) There exist $a_{i j}, b_{i j}>0$ and $\beta_{i j} \in(0,1)$ such that

$$
\begin{gathered}
\qquad\left|I_{i j}(t)\right| \leq a_{i j}+b_{i j}|t|^{\alpha \beta_{i j}} \\
\text { for every } t \in R, i=1,2, \ldots, N ; j=1,2, \ldots, p .
\end{gathered}
$$

Then impulsive problem (1.1) has at least one weak solution.

Remark 1.2 Theorem 1.2 generalizes Theorem B, which is a special case of our Theorem 1.2 corresponding to $G(x) \equiv 0$.

Example 1.2 Let $N=3, p=1$. Consider the following impulsive problem:

$$
\left\{\begin{array}{l}
\ddot{u}(t)=\nabla F(t, u(t)), \quad \text { a.e. } t \in[0, T], \\
u(0)-u(T)=\dot{u}(0)-\dot{u}(T)=0, \\
\Delta \dot{u}^{i}\left(t_{1}\right)=I_{i 1}\left(u^{i}\left(t_{1}\right)\right), \quad i=1,2,3
\end{array}\right.
$$

where

$$
\begin{aligned}
& F(t, x)=\left(\frac{T}{3}-t\right)|x|^{\frac{4}{3}}-\frac{\lambda}{2}\left|x_{1}\right|^{2}+(h(t), x), \\
& h \in L^{1}\left(0, T ; R^{3}\right), \quad I_{i 1}(t)=-t^{\frac{1}{9}} \quad(i=1,2,3) .
\end{aligned}
$$

Take

$$
G(x)=-\frac{\lambda}{2}\left|x_{1}\right|^{2}, \quad x=\left(x^{1}, x^{2}, x^{3}\right) \in R^{3},
$$

which is bounded from above, and

$$
H(t, x)=\left(\frac{T}{3}-t\right)|x|^{\frac{4}{3}}+(h(t), x)
$$

$\alpha=\frac{1}{3}, f(t)=\frac{4}{3}\left(\frac{T}{3}-t\right), g(t)=|h(t)|, a_{i 1}=b_{i 1}=1, \beta_{i 1}=\frac{1}{3}(i=1,2,3)$. Then all the conditions of Theorem 1.2 are satisfied. According to Theorem 1.2, the above problem has at least one weak solution. However, $F$ does not satisfy the condition (h4) in Theorem B. Therefore, our result improves and generalizes Theorem $\mathrm{B}$.

Theorem 1.3 Suppose that $F(t, x)=H(t, x)+G(x)$ and $I_{i j}(t)$ satisfy the assumptions (A), (H1), (H2), (H7) and (H8). Furthermore, assume that

$$
\lim _{|x| \rightarrow+\infty}|x|^{-2 \alpha} F(t, x)=-\infty
$$

uniformly for all $t \in[0, T]$.

Then impulsive problem (1.1) has at least one weak solution. 
Example 1.3 Let $N=3, p=1$. Consider the following impulsive problem:

$$
\left\{\begin{array}{l}
\ddot{u}(t)=\nabla F(t, u(t)), \quad \text { a.e. } t \in[0, T] \\
u(0)-u(T)=\dot{u}(0)-\dot{u}(T)=0 \\
\Delta \dot{u}^{i}\left(t_{1}\right)=I_{i 1}\left(u^{i}\left(t_{1}\right)\right), \quad i=1,2,3
\end{array}\right.
$$

where

$$
F(t, x)=-|x|^{1+\alpha}-\frac{\lambda}{2}\left|x_{1}\right|^{2}, \quad I_{i 1}(t)=-t^{\frac{\alpha}{3}} \quad(i=1,2,3), 0<\alpha<1,0<\lambda<\frac{4 \pi^{2}}{T^{2}} .
$$

Take

$$
H(t, x)=-|x|^{1+\alpha}, \quad G(x)=-\frac{\lambda}{2}\left|x_{1}\right|^{2}, \quad x=\left(x^{1}, x^{2}, x^{3}\right) \in R^{3} .
$$

Then all the conditions of Theorem 1.3 are satisfied. According to Theorem 1.3, the above problem has at least one weak solution. However, $F(t, x)$ is neither superquadratic in $X$ nor subquadratic in $X$.

\section{Preliminaries}

Let $H_{T}^{1}=\left\{u:[0, T] \rightarrow R^{N}: u\right.$ is absolutely continuous, $u(0)=u(T)$ and $\left.\dot{u} \in L^{2}\left(0, T ; R^{N}\right)\right\}$ with the inner product

$$
(u, v)=\int_{0}^{T}(u(t), v(t)) d t+\int_{0}^{T}(\dot{u}(t), \dot{v}(t)) d t, \quad \forall u, v \in H_{T}^{1},
$$

inducing the norm

$$
\|u\|=\left(\int_{0}^{T}|u(t)|^{2} d t+\int_{0}^{T}|\dot{u}(t)|^{2} d t\right)^{\frac{1}{2}}, \quad \forall u \in H_{T}^{1} .
$$

The corresponding functional $\phi$ on $H_{T}^{1}$ given by

$$
\phi(u)=\frac{1}{2} \int_{0}^{T}|\dot{u}(t)|^{2} d t+\int_{0}^{T} F(t, u(t)) d t+\sum_{j=1}^{p} \sum_{i=1}^{N} \int_{0}^{u^{i}\left(t_{j}\right)} I_{i j}(t) d t
$$

is continuously differentiable and weakly lower semi-continuous on $H_{T}^{1}$. For the sake of convenience, we denote

$$
\phi(u)=\phi_{1}(u)+\phi_{2}(u),
$$

where

$$
\phi_{1}(u)=\frac{1}{2} \int_{0}^{T}|\dot{u}(t)|^{2} d t+\int_{0}^{T} F(t, u(t)) d t
$$

and

$$
\phi_{2}(u)=\sum_{j=1}^{p} \sum_{i=1}^{N} \int_{0}^{u^{i}\left(t_{j}\right)} I_{i j}(t) d t
$$


For any $u, v \in H_{T}^{1}$, we have

$$
\begin{aligned}
\left\langle\phi^{\prime}(u), v\right\rangle= & \int_{0}^{T}(\dot{u}(t), \dot{v}(t)) d t+\sum_{j=1}^{p} \sum_{i=1}^{N} I_{i j}\left(u^{i}\left(t_{j}\right)\right) v^{i}\left(t_{j}\right) \\
& +\int_{0}^{T}(\nabla F(t, u(t)), v(t)) d t .
\end{aligned}
$$

Definition 2.1 We say that a function $u \in H_{T}^{1}$ is a weak solution of (1.1) if the identity

$$
\int_{0}^{T}(\dot{u}(t), \dot{v}(t)) d t+\sum_{j=1}^{p} \sum_{i=1}^{N} I_{i j}\left(u^{i}\left(t_{j}\right)\right) v^{i}\left(t_{j}\right)+\int_{0}^{T}(\nabla F(t, u(t)), v(t)) d t=0
$$

holds for any $v \in H_{T}^{1}$.

It is well known that the solutions of impulsive problem (1.1) correspond to the critical point of $\phi$.

Definition 2.2 [1] Let $X$ be a Banach space, $\phi \in C^{1}(X, R)$ and $c \in R$.

(1) $\phi$ is said to satisfy the $(P S)_{c}$-condition on $X$ if the existence of a sequence $\left\{x_{n}\right\} \subseteq X$ such that $\phi\left(x_{n}\right) \rightarrow c$ and $\phi^{\prime}\left(x_{n}\right) \rightarrow 0$ as $n \rightarrow \infty$ implies that $c$ is a critical value of $\phi$.

(2) $\phi$ is said to satisfy the P.S. condition on $X$ if any sequence $\left\{x_{n}\right\} \subseteq X$ for which $\phi\left(x_{n}\right)$ is bounded and $\phi^{\prime}\left(x_{n}\right) \rightarrow 0$ as $n \rightarrow \infty$ possesses a convergent subsequence in $X$.

Remark 2.1 It is clear that the P.S. condition implies the $(P S)_{c}$-condition for each $c \in R$.

Lemma 2.1 [1] If $\phi$ is weakly lower semi-continuous on a reflexive Banach space $X$ (i.e., if $u_{k} \rightarrow u$, then $\left.\liminf _{k \rightarrow \infty} \phi\left(u_{k}\right) \geq \phi(u)\right)$ and has a bounded minimizing sequence, then $\phi$ has a minimum on $X$.

Remark 2.2 The existence of a bounded minimizing sequence will be in particular ensured when $\phi$ is coercive, i.e., such that

$$
\phi(u) \rightarrow+\infty \quad \text { if }\|u\| \rightarrow+\infty
$$

Lemma 2.2 [1] Let $X$ be a Banach space and let $\phi \in C^{1}(X, R)$. Assume that $X$ splits into a direct sum of closed subspaces $X=X^{-} \oplus X^{+}$with $\operatorname{dim} X^{-}<\infty$ and

$$
\sup _{S_{R}^{-}} \phi<\inf _{X^{+}} \phi
$$

where

$$
S_{R}^{-}=\left\{u \in X^{-}:\|u\|=R\right\} .
$$

Let

$$
B_{R}^{-}=\left\{u \in X^{-}:\|u\| \leq R\right\}, \quad M=\left\{h \in C\left(B_{R}^{-}, X\right): h(s)=s \text { if } s \in B_{R}^{-}\right\}
$$


and

$$
c=\inf _{h \in M} \max _{s \in B_{R}^{-}} \phi(h(s)) .
$$

Then if $\phi$ satisfies the $(P S)_{c}$-condition, $c$ is a critical value of $\phi$.

Lemma 2.3 [1] If the sequence $\left\{u_{k}\right\}$ converges weakly to $u$ in $H_{T}^{1}$, then $\left\{u_{k}\right\}$ converges uniformly to $u$ on $[0, T]$.

Lemma 2.4 [1] If $u \in H_{T}^{1}$ and $\int_{0}^{T} u(t) d t=0$, then

$$
\int_{0}^{T}|u(t)|^{2} d t \leq \frac{T^{2}}{4 \pi^{2}} \int_{0}^{T}|\dot{u}(t)|^{2} d t \quad \text { (Wirtinger's inequality) }
$$

and

$$
\|u\|_{\infty}^{2} \leq \frac{T}{12} \int_{0}^{T}|\dot{u}(t)|^{2} d t \quad \text { (Sobolev's inequality) }
$$

Lemma 2.5 There exists $C_{*}>0$ such that if $u \in H_{T}^{1}$, then

$$
\|u\|_{\infty} \leq C_{*}\|u\|
$$

Moreover, if $\int_{0}^{T} u(t) d t=0$, then

$$
\|u\|_{\infty} \leq C_{*}\|\dot{u}\|_{L^{2}}
$$

where $\|u\|_{\infty}=\max _{t \in[0, T]}|u(t)|$ and $\|u\|_{L^{2}}=\left(\int_{0}^{T}|u(t)|^{2} d t\right)^{\frac{1}{2}}$.

\section{Proof of main results}

For $u \in H_{T}^{1}$, let $\bar{u}=\frac{1}{T} \int_{0}^{T} u(t) d t$ and $\tilde{u}(t)=u(t)-\bar{u}$.

Proof of Theorem 1.1 It follows from (H1) and Sobolev's inequality that

$$
\begin{aligned}
& \left|\int_{0}^{T}[H(t, u(t))-H(t, \bar{u})] d t\right| \\
& \quad=\left|\int_{0}^{T} \int_{0}^{1}(\nabla H(t, \bar{u}+s \tilde{u}(t)), \tilde{u}(t)) d s d t\right| \\
& \quad \leq \int_{0}^{T} \int_{0}^{1} f(t)|\bar{u}+s \tilde{u}(t)|^{\alpha}|\tilde{u}(t)| d s d t+\int_{0}^{T} \int_{0}^{1} g(t)|\tilde{u}(t)| d s d t \\
& \quad \leq \int_{0}^{T} 2 f(t)\left(|\bar{u}|^{\alpha}+|\tilde{u}(t)|^{\alpha}\right)|\tilde{u}(t)| d t+\int_{0}^{T} g(t)|\tilde{u}(t)| d t \\
& \quad \leq 2\left(|\bar{u}|^{\alpha}+\|\tilde{u}\|_{\infty}^{\alpha}\right)\|\tilde{u}\|_{\infty} \int_{0}^{T} f(t) d t+\|\tilde{u}\|_{\infty} \int_{0}^{T} g(t) d t \\
& \quad \leq \frac{3\left(4 \pi^{2}-\lambda T^{2}\right)}{4 \pi^{2} T}\|\tilde{u}\|_{\infty}^{2}+\frac{4 \pi^{2} T}{3\left(4 \pi^{2}-\lambda T^{2}\right)}|\bar{u}|^{2 \alpha}\left(\int_{0}^{T} f(t) d t\right)^{2}
\end{aligned}
$$




$$
\begin{aligned}
& +2\|\tilde{u}\|_{\infty}^{\alpha+1} \int_{0}^{T} f(t) d t+\|\tilde{u}\|_{\infty} \int_{0}^{T} g(t) d t \\
\leq & \frac{4 \pi^{2}-\lambda T^{2}}{16 \pi^{2}} \int_{0}^{T}|\dot{u}(t)|^{2} d t+C_{1}|\bar{u}|^{2 \alpha}+C_{2}\left(\int_{0}^{T}|\dot{u}(t)|^{2} d t\right)^{\frac{\alpha+1}{2}} \\
& +C_{3}\left(\int_{0}^{T}|\dot{u}(t)|^{2} d t\right)^{\frac{1}{2}}
\end{aligned}
$$

for all $u \in H_{T}^{1}$ and some positive constants $C_{1}, C_{2}$ and $C_{3}$. By (H2) and Wirtinger's inequality, we have

$$
\begin{aligned}
\int_{0}^{T}[G(u(t))-G(\bar{u})] d t & =\int_{0}^{T} \int_{0}^{1}(\nabla G(\bar{u}+s \tilde{u}(t))-\nabla G(\bar{u}), \tilde{u}(t)) d s d t \\
& =\int_{0}^{T} \int_{0}^{1} \frac{1}{s}(\nabla G(\bar{u}+s \tilde{u}(t))-\nabla G(\bar{u}), s \tilde{u}(t)) d s d t \\
& \geq \int_{0}^{T} \int_{0}^{1}(-\lambda) s|\tilde{u}(t)|^{2} d s d t \\
& =-\frac{\lambda}{2} \int_{0}^{T}|\tilde{u}(t)|^{2} d t \\
& \geq-\frac{\lambda T^{2}}{8 \pi^{2}} \int_{0}^{T}|\dot{u}(t)|^{2} d t
\end{aligned}
$$

for all $u \in H_{T}^{1}$.

From (H4), we obtain

$$
\phi_{2}(u) \geq 0
$$

for all $u \in H_{T}^{1}$. Therefore we have

$$
\begin{aligned}
\phi(u)= & \frac{1}{2} \int_{0}^{T}|\dot{u}(t)|^{2} d t+\int_{0}^{T}[F(t, u(t))-F(t, \bar{u})] d t+\int_{0}^{T} F(t, \bar{u}) d t+\phi_{2}(u) \\
= & \frac{1}{2} \int_{0}^{T}|\dot{u}(t)|^{2} d t+\int_{0}^{T}[H(t, u(t))-H(t, \bar{u})] d t+\int_{0}^{T}[G(u(t))-G(\bar{u})] d t \\
& +\int_{0}^{T} F(t, \bar{u}) d t+\phi_{2}(u) \\
\geq & \frac{1}{2} \int_{0}^{T}|\dot{u}(t)|^{2} d t+\int_{0}^{T}[H(t, u(t))-H(t, \bar{u})] d t+\int_{0}^{T}[G(u(t))-G(\bar{u})] d t \\
& +\int_{0}^{T} F(t, \bar{u}) d t \\
\geq & \frac{1}{2} \int_{0}^{T}|\dot{u}(t)|^{2} d t-\frac{4 \pi^{2}-\lambda T^{2}}{16 \pi^{2}} \int_{0}^{T}|\dot{u}(t)|^{2} d t-C_{1}|\bar{u}|^{2 \alpha} \\
& -C_{2}\left(\int_{0}^{T}|\dot{u}(t)|^{2} d t\right)^{\frac{\alpha+1}{2}}-C_{3}\left(\int_{0}^{T}|\dot{u}(t)|^{2} d t\right)^{\frac{1}{2}}-\frac{\lambda T^{2}}{8 \pi^{2}} \int_{0}^{T}|\dot{u}(t)|^{2} d t \\
& +\int_{0}^{T} F(t, \bar{u}) d t
\end{aligned}
$$




$$
\begin{aligned}
= & \frac{4 \pi^{2}-\lambda T^{2}}{16 \pi^{2}} \int_{0}^{T}|\dot{u}(t)|^{2} d t+|\bar{u}|^{2 \alpha}\left(|\bar{u}|^{-2 \alpha} \int_{0}^{T} F(t, \bar{u}) d t-C_{1}\right) \\
& -C_{2}\left(\int_{0}^{T}|\dot{u}(t)|^{2} d t\right)^{\frac{\alpha+1}{2}}-C_{3}\left(\int_{0}^{T}|\dot{u}(t)|^{2} d t\right)^{\frac{1}{2}}
\end{aligned}
$$

for all $u \in H_{T}^{1}$. As $\|u\| \rightarrow \infty$ if and only if $\left(|\bar{u}|^{2}+\|\dot{u}(t)\|_{L^{2}}^{2}\right)^{\frac{1}{2}} \rightarrow \infty$, (3.3) and (H3) imply that

$$
\phi(u) \rightarrow+\infty \quad \text { as }\|u\| \rightarrow \infty
$$

By Lemma 2.1 and Remark 2.1, $\phi$ has a minimum point on $H_{T}^{1}$, which is a critical point of $\phi$. Therefore, we complete the proof of Theorem 1.1.

Lemma 3.1 Assume that the conditions of Theorem 1.2 hold. Then $\phi$ satisfies the P.S. condition.

Proof Let $\left\{\phi\left(u_{n}\right)\right\}$ be bounded and $\phi^{\prime}\left(u_{n}\right) \rightarrow 0$ as $n \rightarrow \infty$. From (H1) and Lemma 2.4, we have

$$
\begin{aligned}
\mid \int_{0}^{T} & \left(\nabla H\left(t, u_{n}(t)\right), \tilde{u}_{n}(t)\right) d t \mid \\
\leq & \int_{0}^{T}\left|\nabla H\left(t, u_{n}(t)\right)\right|\left|\tilde{u}_{n}(t)\right| d t \\
\leq & \int_{0}^{T} f(t)\left|\bar{u}_{n}+\tilde{u}(t)\right|^{\alpha}\left|\tilde{u}_{n}(t)\right| d t+\int_{0}^{T} g(t)\left|\tilde{u}_{n}(t)\right| d t \\
\leq & 2\left(\left|\bar{u}_{n}\right|^{\alpha}+\left\|\tilde{u}_{n}\right\|_{\infty}^{\alpha}\right)\left\|\tilde{u}_{n}\right\|_{\infty} \int_{0}^{T} f(t) d t+\left\|\tilde{u}_{n}\right\|_{\infty} \int_{0}^{T} g(t) d t \\
\leq & \frac{3\left(4 \pi^{2}-A T^{2}\right)}{2 \pi^{2} T}\left\|\tilde{u}_{n}\right\|_{\infty}^{2}+\frac{2 \pi^{2} T}{3\left(4 \pi^{2}-A T^{2}\right)}\left|\bar{u}_{n}\right|^{2 \alpha}\left(\int_{0}^{T} f(t) d t\right)^{2} \\
& +2\left\|\tilde{u}_{n}\right\|_{\infty}^{\alpha+1} \int_{0}^{T} f(t) d t+\left\|\tilde{u}_{n}\right\|_{\infty} \int_{0}^{T} g(t) d t \\
\leq & \frac{4 \pi^{2}-A T^{2}}{8 \pi^{2}} \int_{0}^{T}\left|\dot{u}_{n}(t)\right|^{2} d t+C_{4}\left|\bar{u}_{n}\right|^{2 \alpha} \\
& +C_{5}\left(\int_{0}^{T}\left|\dot{u}_{n}(t)\right|^{2} d t\right)^{\frac{\alpha+1}{2}}+C_{6}\left(\int_{0}^{T}\left|\dot{u}_{n}(t)\right|^{2} d t\right)^{\frac{1}{2}}
\end{aligned}
$$

for all large $n$ and some positive constants $C_{4}, C_{5}$ and $C_{6}$. It follows from (H5) and Lemma 2.4 that

$$
\begin{aligned}
\left|\int_{0}^{T}\left(\nabla G\left(u_{n}(t)\right), \tilde{u}_{n}(t)\right) d t\right| & =\left|\int_{0}^{T}\left(\nabla G\left(u_{n}(t)\right)-\nabla G\left(\bar{u}_{n}\right), \tilde{u}_{n}(t)\right) d t\right| \\
& \leq \int_{0}^{T}\left|\nabla G\left(u_{n}(t)\right)-\nabla G\left(\bar{u}_{n}\right)\right|\left|\tilde{u}_{n}(t)\right| d t \\
& \leq \int_{0}^{T}\left(A\left|u_{n}(t)-\bar{u}_{n}\right|+B\right)\left|\tilde{u}_{n}(t)\right| d t
\end{aligned}
$$




$$
\begin{aligned}
& =A \int_{0}^{T}\left|\tilde{u}_{n}(t)\right|^{2} d t+B \int_{0}^{T}\left|\tilde{u}_{n}(t)\right| d t \\
& \leq A \int_{0}^{T}\left|\tilde{u}_{n}(t)\right|^{2} d t+B \sqrt{T}\left(\int_{0}^{T}\left|\tilde{u}_{n}(t)\right|^{2} d t\right)^{\frac{1}{2}} \\
& \leq \frac{A T^{2}}{4 \pi^{2}} \int_{0}^{T}\left|\dot{u}_{n}(t)\right|^{2} d t+\frac{B T \sqrt{T}}{2 \pi}\left(\int_{0}^{T}\left|\dot{u}_{n}(t)\right|^{2} d t\right)^{\frac{1}{2}} .
\end{aligned}
$$

By (3.4), (3.5), (H8) and Young's inequality, we have

$$
\begin{aligned}
& \left\|\tilde{u}_{n}\right\| \geq\left\langle\phi^{\prime}\left(u_{n}\right), \tilde{u}_{n}\right\rangle \\
& =\int_{0}^{T}\left|\dot{u}_{n}(t)\right|^{2} d t+\int_{0}^{T}\left(\nabla F\left(t, u_{n}(t)\right), \tilde{u}_{n}(t)\right) d t+\sum_{j=1}^{p} \sum_{i=1}^{N} I_{i j}\left(u_{n}^{i}(t)\right) \tilde{u}_{n}^{i}(t) \\
& =\int_{0}^{T}\left|\dot{u}_{n}(t)\right|^{2} d t+\int_{0}^{T}\left(\nabla H\left(t, u_{n}(t)\right), \tilde{u}_{n}(t)\right) d t+\int_{0}^{T}\left(\nabla G\left(u_{n}(t)\right), \tilde{u}_{n}(t)\right) d t \\
& +\sum_{j=1}^{p} \sum_{i=1}^{N} I_{i j}\left(u_{n}^{i}(t)\right) \tilde{u}_{n}^{i}(t) \\
& \geq \frac{4 \pi^{2}-A T^{2}}{8 \pi^{2}} \int_{0}^{T}\left|\dot{u}_{n}(t)\right|^{2} d t-C_{4}\left|\bar{u}_{n}\right|^{2 \alpha}-C_{5}\left(\int_{0}^{T}\left|\dot{u}_{n}(t)\right|^{2} d t\right)^{\frac{\alpha+1}{2}} \\
& -\left(C_{6}+\frac{B T \sqrt{T}}{2 \pi}\right)\left(\int_{0}^{T}\left|\dot{u}_{n}(t)\right|^{2} d t\right)^{\frac{1}{2}}-\sum_{j=1}^{p} \sum_{i=1}^{N}\left(a_{i j}+b_{i j}\left|u_{n}^{i}(t)\right|^{\alpha \beta_{i j}}\right)\left|\tilde{u}_{n}^{i}(t)\right| \\
& \geq \frac{4 \pi^{2}-A T^{2}}{8 \pi^{2}} \int_{0}^{T}\left|\dot{u}_{n}(t)\right|^{2} d t-C_{4}\left|\bar{u}_{n}\right|^{2 \alpha}-C_{5}\left(\int_{0}^{T}\left|\dot{u}_{n}(t)\right|^{2} d t\right)^{\frac{\alpha+1}{2}} \\
& -\left(C_{6}+\frac{B T \sqrt{T}}{2 \pi}\right)\left(\int_{0}^{T}\left|\dot{u}_{n}(t)\right|^{2} d t\right)^{\frac{1}{2}}-a p N\left\|\tilde{u}_{n}\right\|_{\infty} \\
& -b \sum_{j=1}^{p} \sum_{i=1}^{N} 2\left(\left|\bar{u}_{n}\right|^{\alpha \beta_{i j}}+\left\|\tilde{u}_{n}\right\|_{\infty}^{\alpha \beta_{i j}}\right)\left\|\tilde{u}_{n}\right\|_{\infty} \\
& \geq \frac{4 \pi^{2}-A T^{2}}{8 \pi^{2}} \int_{0}^{T}\left|\dot{u}_{n}(t)\right|^{2} d t-C_{4}\left|\bar{u}_{n}\right|^{2 \alpha}-C_{5}\left(\int_{0}^{T}\left|\dot{u}_{n}(t)\right|^{2} d t\right)^{\frac{\alpha+1}{2}} \\
& -\left(C_{6}+\frac{B T \sqrt{T}}{2 \pi}\right)\left(\int_{0}^{T}\left|\dot{u}_{n}(t)\right|^{2} d t\right)^{\frac{1}{2}}-a p N \sqrt{\frac{T}{12}}\left(\int_{0}^{T}\left|\dot{u}_{n}(t)\right|^{2} d t\right)^{\frac{1}{2}} \\
& -b \sum_{j=1}^{p} \sum_{i=1}^{N} \beta_{i j}\left|\bar{u}_{n}\right|^{2 \alpha}-2 b \sum_{j=1}^{p} \sum_{i=1}^{N} \frac{2-\beta_{i j}}{2}\left\|\tilde{u}_{n}\right\|_{\infty}^{\frac{2}{2-\beta_{i j}}} \\
& -2 b \sum_{j=1}^{p} \sum_{i=1}^{N}\left\|\tilde{u}_{n}\right\|_{\infty}^{\alpha \beta_{i j}+1} \\
& \geq \frac{4 \pi^{2}-A T^{2}}{8 \pi^{2}} \int_{0}^{T}\left|\dot{u}_{n}(t)\right|^{2} d t-C_{4}\left|\bar{u}_{n}\right|^{2 \alpha}-C_{5}\left(\int_{0}^{T}\left|\dot{u}_{n}(t)\right|^{2} d t\right)^{\frac{\alpha+1}{2}} \\
& -\left(C_{6}+\frac{B T \sqrt{T}}{2 \pi}\right)\left(\int_{0}^{T}\left|\dot{u}_{n}(t)\right|^{2} d t\right)^{\frac{1}{2}}-a p N \sqrt{\frac{T}{12}}\left(\int_{0}^{T}\left|\dot{u}_{n}(t)\right|^{2} d t\right)^{\frac{1}{2}}
\end{aligned}
$$




$$
\begin{aligned}
& -b \sum_{j=1}^{p} \sum_{i=1}^{N} \beta_{i j}\left|\bar{u}_{n}\right|^{2 \alpha}-b \sum_{j=1}^{p} \sum_{i=1}^{N}\left(2-\beta_{i j}\right)\left(\frac{T}{12} \int_{0}^{T}\left|\dot{u}_{n}(t)\right|^{2} d t\right)^{\frac{1}{2-\beta_{i j}}} \\
& -2 b \sum_{j=1}^{p} \sum_{i=1}^{N}\left(\frac{T}{12} \int_{0}^{T}\left|\dot{u}_{n}(t)\right|^{2} d t\right)^{\frac{\alpha \beta_{i j}+1}{2}}
\end{aligned}
$$

where $a=\max \left\{a_{i j}, i=1,2, \ldots, N ; j=1,2, \ldots, p\right\}, b=\max \left\{b_{i j}, i=1,2, \ldots, N ; j=1,2, \ldots, p\right\}$. From Wirtinger's inequality, we obtain

$$
\int_{0}^{T}\left|\dot{u}_{n}(t)\right|^{2} d t \leq\left\|\tilde{u}_{n}\right\|^{2} \leq\left(1+\frac{T^{2}}{4 \pi^{2}}\right) \int_{0}^{T}\left|\dot{u}_{n}(t)\right|^{2} d t
$$

The inequalities (3.6) and (3.7) imply that

$$
C_{7}\left|\bar{u}_{n}\right|^{\alpha} \geq\left(\int_{0}^{T}\left|\dot{u}_{n}(t)\right|^{2} d t\right)^{\frac{1}{2}}-C_{8}
$$

for all large $n$ and some positive constants $C_{7}$ and $C_{8}$. It follows from (H5), CauchySchwarz's inequality and Wirtinger's inequality that

$$
\begin{aligned}
\left|\int_{0}^{T}\left[G\left(u_{n}(t)\right)-G\left(\bar{u}_{n}\right)\right] d t\right| & =\left|\int_{0}^{T} \int_{0}^{1}\left(\nabla G\left(\bar{u}_{n}+s \tilde{u}_{n}(t)\right)-\nabla G\left(\bar{u}_{n}\right), \tilde{u}_{n}(t)\right) d s d t\right| \\
& \leq \int_{0}^{T} \int_{0}^{1}\left(A s\left|\tilde{u}_{n}(t)\right|+B\right)\left|\tilde{u}_{n}(t)\right| d s d t \\
& =\frac{A}{2} \int_{0}^{T}\left|\tilde{u}_{n}(t)\right|^{2} d t+B \int_{0}^{T}\left|\tilde{u}_{n}(t)\right| d t \\
& \leq \frac{A}{2} \int_{0}^{T}\left|\tilde{u}_{n}(t)\right|^{2} d t+B \sqrt{T}\left(\int_{0}^{T}\left|\tilde{u}_{n}(t)\right|^{2} d t\right)^{\frac{1}{2}} \\
& \leq \frac{A T^{2}}{8 \pi^{2}} \int_{0}^{T}\left|\dot{u}_{n}(t)\right|^{2} d t+\frac{B T \sqrt{T}}{2 \pi}\left(\int_{0}^{T}\left|\dot{u}_{n}(t)\right|^{2} d t\right)^{\frac{1}{2}} .
\end{aligned}
$$

Like in the proof of Theorem 1.1, we get

$$
\begin{aligned}
\left|\int_{0}^{T}\left[H\left(t, u_{n}(t)\right)-H\left(t, \bar{u}_{n}\right)\right] d t\right| \leq & \frac{4 \pi^{2}-A T^{2}}{16 \pi^{2}} \int_{0}^{T}\left|\dot{u}_{n}(t)\right|^{2} d t+C_{1}\left|\bar{u}_{n}\right|^{2 \alpha} \\
& +C_{2}\left(\int_{0}^{T}\left|\dot{u}_{n}(t)\right|^{2} d t\right)^{\frac{\alpha+1}{2}} \\
& +C_{3}\left(\int_{0}^{T}\left|\dot{u}_{n}(t)\right|^{2} d t\right)^{\frac{1}{2}} .
\end{aligned}
$$

From (H7), we have

$$
\phi_{2}(u) \leq 0
$$


for all $u \in H_{T}^{1}$. Since $\left\{\phi\left(u_{n}\right)\right\}$ is bounded, from (3.9) and (3.10), there exists a constant $C$ such that

$$
\begin{aligned}
C \leq & \phi\left(u_{n}\right) \\
= & \frac{1}{2} \int_{0}^{T}\left|\dot{u}_{n}(t)\right|^{2} d t+\int_{0}^{T}\left[F\left(t, u_{n}(t)\right)-F\left(t, \bar{u}_{n}\right)\right] d t+\int_{0}^{T} F\left(t, \bar{u}_{n}\right) d t+\phi_{2}\left(u_{n}\right) \\
\leq & \frac{1}{2} \int_{0}^{T}\left|\dot{u}_{n}(t)\right|^{2} d t+\int_{0}^{T}\left[H\left(t, u_{n}(t)\right)-H\left(t, \bar{u}_{n}\right)\right] d t \\
& +\int_{0}^{T}\left[G\left(u_{n}(t)\right)-G\left(\bar{u}_{n}\right)\right] d t+\int_{0}^{T} F\left(t, \bar{u}_{n}\right) d t \\
\leq & \frac{12 \pi^{2}+A T^{2}}{16 \pi^{2}} \int_{0}^{T}\left|\dot{u}_{n}(t)\right|^{2} d t+C_{1}\left|\bar{u}_{n}\right|^{2 \alpha}+C_{2}\left(\int_{0}^{T}\left|\dot{u}_{n}(t)\right|^{2} d t\right)^{\frac{\alpha+1}{2}} \\
& +C_{3}\left(\int_{0}^{T}\left|\dot{u}_{n}(t)\right|^{2} d t\right)^{\frac{1}{2}}+\frac{B T \sqrt{T}}{2 \pi}\left(\left|\dot{u}_{n}(t)\right|^{2} d t\right)^{\frac{1}{2}}+\int_{0}^{T} F\left(t, \bar{u}_{n}\right) d t \\
\leq & \left|\bar{u}_{n}\right|^{2 \alpha}\left(\left|\bar{u}_{n}\right|^{-2 \alpha} \int_{0}^{T} F\left(t, \bar{u}_{n}\right) d t+C_{0}\right)
\end{aligned}
$$

for all large $n$ and some constant $C_{0}$. By the above inequality and (H6), we know that $\left\{\left|\bar{u}_{n}\right|\right\}$ is bounded. In fact, if not, without loss of generality, we may assume that $\left|\bar{u}_{n}\right| \rightarrow \infty$ as $n \rightarrow \infty$. Then, from (3.8) and the above inequality, we have

$$
\lim _{n \rightarrow \infty} \inf \left|\bar{u}_{n}\right|^{-2 \alpha} \int_{0}^{T} F\left(t, \bar{u}_{n}\right) d t>-\infty
$$

which contradicts (H6). Hence $\left\{\left|\bar{u}_{n}\right|\right\}$ is bounded. Furthermore, $\left\{u_{n}\right\}$ is bounded from (3.7) and (3.8). Hence, there exists a subsequence of $u_{n}$ defined by $u_{n}^{*}$ such that

$$
u_{n}^{*} \rightarrow u \quad \text { in } H_{T}^{1} \text {. }
$$

By Lemma 2.3, we have

$$
u_{n}^{*} \rightarrow u \quad \text { in } C[0, T]
$$

On the other hand, we get

$$
\begin{aligned}
\left\langle\phi^{\prime}\left(u_{n}^{*}\right)-\phi^{\prime}(u), u_{n}^{*}-u\right\rangle= & \int_{0}^{T}\left|\dot{u}_{n}^{*}(t)-\dot{u}(t)\right|^{2} d t \\
& +\int_{0}^{T}\left(\nabla F\left(t, u_{n}^{*}(t)\right)-\nabla F(t, u(t)), u_{n}^{*}(t)-u(t)\right) d t \\
& +\sum_{j=1}^{p} \sum_{i=1}^{N}\left[I_{i j}\left(u_{n}^{* i}\left(t_{j}\right)\right)-I_{i j}\left(u^{i}\left(t_{j}\right)\right)\right]\left(u_{n}^{* i}\left(t_{j}\right)-u^{i}\left(t_{j}\right)\right) .
\end{aligned}
$$

It follows from the above equality, (A) and the continuity of $I_{i j}$ that

$$
u_{n}^{*} \rightarrow u \quad \text { in } H_{T}^{1}
$$

Thus, we conclude that $\phi$ satisfies the P.S. condition. 
Now, we give the proof of our Theorem 1.2.

Proof of Theorem 1.2 Let $W$ be the subspace of $H_{T}^{1}$ given by

$$
W=\left\{u \in H_{T}^{1} \mid \bar{u}=0\right\} .
$$

Then $H_{T}^{1}=W \oplus R^{N}$. Firstly, we show that

$$
\phi(u) \rightarrow+\infty \quad \text { as } u \in W,\|u\| \rightarrow \infty .
$$

In fact, for $u \in W$, then $\bar{u}=0$, by the proof of Theorem 1.1, we have

$$
\begin{aligned}
\left|\int_{0}^{T}[H(t, u(t))-H(t, 0)] d t\right| \leq & \frac{4 \pi^{2}-A T^{2}}{16 \pi^{2}} \int_{0}^{T}|\dot{u}(t)|^{2} d t+C_{2}\left(\int_{0}^{T}|\dot{u}(t)|^{2} d t\right)^{\frac{\alpha+1}{2}} \\
& +C_{3}\left(\int_{0}^{T}|\dot{u}(t)|^{2} d t\right)^{\frac{1}{2}}
\end{aligned}
$$

Like in the proof of Lemma 3.1, we obtain

$$
\begin{aligned}
\left|\int_{0}^{T}[G(u(t))-G(0)] d t\right| & =\left|\int_{0}^{T} \int_{0}^{1}(\nabla G(s u)-\nabla G(0), u) d s d t\right| \\
& \leq \frac{A}{2} \int_{0}^{T}|u(t)|^{2} d t+B \int_{0}^{T}|u(t)| d t \\
& \leq \frac{A T^{2}}{8 \pi^{2}} \int_{0}^{T}\left|\dot{u}_{n}(t)\right|^{2} d t+\frac{B T \sqrt{T}}{2 \pi}\left(\int_{0}^{T}\left|\dot{u}_{n}(t)\right|^{2} d t\right)^{\frac{1}{2}} .
\end{aligned}
$$

By (H8) and Lemma 2.5, we find

$$
\begin{aligned}
\left|\phi_{2}(u)\right| & =\left|\sum_{j=1}^{p} \sum_{i=1}^{N} \int_{0}^{u^{i}\left(t_{j}\right)} I_{i j}(t) d t\right| \\
& \leq \sum_{j=1}^{p} \sum_{i=1}^{N} \int_{0}^{u^{i}\left(t_{j}\right)}\left(a_{i j}+b_{i j}|t|^{\alpha \beta_{i j}}\right) d t \\
& \leq a p N\|u\|_{\infty}+b \sum_{j=1}^{p} \sum_{i=1}^{N}\|u\|_{\infty}^{\alpha \beta_{i j}+1} \\
& \leq a p N C_{*}\left(\int_{0}^{T}|\dot{u}(t)|^{2} d t\right)^{\frac{1}{2}}+b \sum_{j=1}^{p} \sum_{i=1}^{N}\left(C_{*}^{2} \int_{0}^{T}|\dot{u}(t)|^{2} d t\right)^{\frac{\alpha \beta_{i j}+1}{2}}
\end{aligned}
$$

for all $u \in W$. By (3.14), (3.15) and (3.16), we have

$$
\begin{aligned}
\phi(u) & =\frac{1}{2} \int_{0}^{T}|\dot{u}(t)|^{2} d t+\int_{0}^{T}[F(t, u(t))-F(t, 0)] d t+\int_{0}^{T} F(t, 0) d t+\phi_{2}(u) \\
& =\frac{1}{2} \int_{0}^{T}|\dot{u}(t)|^{2} d t+\int_{0}^{T}[H(t, u(t))-H(t, 0)] d t+\int_{0}^{T}[G(u(t))-G(0)] d t
\end{aligned}
$$




$$
\begin{aligned}
& +\int_{0}^{T} F(t, 0) d t+\phi_{2}(u) \\
\geq & \left(\frac{4 \pi^{2}-A T^{2}}{16 \pi^{2}}\right) \int_{0}^{T}|\dot{u}(t)|^{2} d t-C_{2}\left(\int_{0}^{T}|\dot{u}(t)|^{2} d t\right)^{\frac{\alpha+1}{2}} \\
& -C_{3}\left(\int_{0}^{T}|\dot{u}(t)|^{2} d t\right)^{\frac{1}{2}}-\frac{B T \sqrt{T}}{2 \pi}\left(\int_{0}^{T}\left|\dot{u}_{n}(t)\right|^{2} d t\right)^{\frac{1}{2}} \\
& -a p N C_{*}\left(\int_{0}^{T}|\dot{u}(t)|^{2} d t\right)^{\frac{1}{2}}-b \sum_{j=1}^{p} \sum_{i=1}^{N}\left(C_{*}^{2} \int_{0}^{T}|\dot{u}(t)|^{2} d t\right)^{\frac{\alpha \beta_{i j}+1}{2}} d t \\
& +\int_{0}^{T} F(t, 0) d t
\end{aligned}
$$

for all $u \in W$.

By Lemma 2.4, we have $\|u\| \rightarrow \infty \Leftrightarrow\|\dot{u}\|_{L^{2}} \rightarrow \infty$ on $W$. Hence (3.13) follows from (3.17).

On the other hand, by (H7), we get

$$
\phi_{2}(u) \leq 0
$$

for all $u \in H_{T}^{1}$. Therefore, from (3.18) and (H6), we obtain

$$
\phi(u)=\int_{0}^{T} F(t, u) d t+\phi_{2}(u) \leq|u|^{2 \alpha}\left(|u|^{-2 \alpha} \int_{0}^{T} F(t, u) d t\right) \rightarrow-\infty
$$

as $|u| \rightarrow \infty$ in $R^{N}$. It follows from Lemma 2.2 and Lemma 3.1 that problem (1.1) has at least one weak solution.

Proof of Theorem 1.3 First we prove that $\phi$ satisfies the P.S. condition. Suppose that $U_{n}$ is a P.S. sequence for $\phi$, that is, $\phi^{\prime}\left(u_{n}\right) \rightarrow 0$ as $n \rightarrow \infty$ and $\phi\left(u_{n}\right)$ is bounded. In a way similar to the proof of Theorem 1.1, we have

$$
\begin{aligned}
\left|\int_{0}^{T}\left(\nabla H\left(t, u_{n}(t)\right), \tilde{n}_{n}(t)\right) d t\right| \leq & \frac{4 \pi^{2}-\lambda T^{2}}{16 \pi^{2}} \int_{0}^{T}\left|\dot{u}_{n}(t)\right|^{2} d t+C_{1}\left|\bar{u}_{n}\right|^{2 \alpha} \\
& +C_{2}\left(\int_{0}^{T}\left|\dot{u}_{n}(t)\right|^{2} d t\right)^{\frac{\alpha+1}{2}}+C_{3}\left(\int_{0}^{T}\left|\dot{u}_{n}(t)\right|^{2} d t\right)^{\frac{1}{2}}
\end{aligned}
$$

and

$$
\int_{0}^{T}\left(\nabla G\left(u_{n}(t)\right), \tilde{u}_{n}(t)\right) d t \geq-\frac{\lambda T^{2}}{4 \pi^{2}} \int_{0}^{T}\left|\dot{u}_{n}(t)\right|^{2} d t
$$

for all $u \in H_{T}^{1}$. Hence we have

$$
\begin{aligned}
\left\|\tilde{u}_{n}\right\| & \geq\left\langle\phi^{\prime}\left(u_{n}\right), \tilde{u}_{n}\right\rangle \\
& =\int_{0}^{T}\left|\dot{u}_{n}(t)\right|^{2} d t+\int_{0}^{T}\left(\nabla F\left(t, u_{n}(t)\right), \tilde{u}_{n}(t)\right) d t+\sum_{j=1}^{p} \sum_{i=1}^{N} I_{i j}\left(u_{n}^{i}(t)\right) \tilde{u}_{n}^{i}(t)
\end{aligned}
$$




$$
\begin{aligned}
= & \int_{0}^{T}\left|\dot{u}_{n}(t)\right|^{2} d t+\int_{0}^{T}\left(\nabla H\left(t, u_{n}(t)\right), \tilde{u}_{n}(t)\right) d t \\
& +\int_{0}^{T}\left(\nabla G\left(u_{n}(t)\right), \tilde{u}_{n}(t)\right) d t+\sum_{j=1}^{p} \sum_{i=1}^{N} I_{i j}\left(u_{n}^{i}(t)\right) \tilde{u}_{n}^{i}(t) \\
\geq & \frac{3\left(4 \pi^{2}-\lambda T^{2}\right)}{16 \pi^{2}} \int_{0}^{T}\left|\dot{u}_{n}(t)\right|^{2} d t-C_{1}\left|\bar{u}_{n}\right|^{2 \alpha}-C_{2}\left(\int_{0}^{T}\left|\dot{u}_{n}(t)\right|^{2} d t\right)^{\frac{\alpha+1}{2}} \\
& -C_{3}\left(\int_{0}^{T}\left|\dot{u}_{n}(t)\right|^{2} d t\right)^{\frac{1}{2}}-a p N \sqrt{\frac{T}{12}}\left(\int_{0}^{T}\left|\dot{u}_{n}(t)\right|^{2} d t\right)^{\frac{1}{2}} \\
& -b \sum_{j=1}^{p} \sum_{i=1}^{N} \beta_{i j}\left|\bar{u}_{n}\right|^{2 \alpha}-b \sum_{j=1}^{p} \sum_{i=1}^{N}\left(2-\beta_{i j}\right)\left(\frac{T}{12} \int_{0}^{T}\left|\dot{u}_{n}(t)\right|^{2} d t\right)^{\frac{1}{2-\beta_{i j}}} \\
& -2 b \sum_{j=1}^{p} \sum_{i=1}^{N}\left(\frac{T}{12} \int_{0}^{T}\left|\dot{u}_{n}(t)\right|^{2} d t\right)^{\frac{\alpha \beta_{i j}+1}{2}} .
\end{aligned}
$$

From (3.7) and the above inequalities, we obtain

$$
C_{9}\left|\bar{u}_{n}\right|^{\alpha} \geq\left(\int_{0}^{T}\left|\dot{u}_{n}(t)\right|^{2} d t\right)^{\frac{1}{2}}-C_{10}
$$

for some positive constants $C_{9}$ and $C_{10}$.

By (H9) there exists $M>0$ such that

$$
|x|^{-2 \alpha} F(t, x) \leq 0
$$

for all $|x| \geq M$ and $t \in[0, T]$, which implies that

$$
F(t, x) \leq 0
$$

for all $|x| \geq M$ and $t \in[0, T]$. It follows from assumption (A) that

$$
F(t, x) \leq a_{0} b(t)
$$

for all $|x| \leq M$ and $t \in[0, T]$, where $a_{0}=\max _{0 \leq s \leq M} a(s)$.

$$
\text { Let } \gamma(t)=a_{0} b(t) \in L^{1}(0, T) \text {, then }
$$

$$
F(t, x) \leq \gamma(t)
$$

for all $x \in R^{N}$ and $t \in[0, T]$.

By the boundedness of $\phi\left(u_{n}\right),(\mathrm{H} 7)$ and (3.19), there exists a constant $C_{11}$ such that

$$
\begin{aligned}
C_{11} \leq \phi\left(u_{n}\right) & =\frac{1}{2} \int_{0}^{T}\left|\dot{u}_{n}(t)\right|^{2} d t+\int_{0}^{T} F\left(t, u_{n}(t)\right) d t+\phi_{2}(u) \\
& \leq \frac{1}{2} \int_{0}^{T}\left|\dot{u}_{n}(t)\right|^{2} d t+\int_{0}^{T} F\left(t, u_{n}(t)\right) d t \leq\left(C_{9}\left|\bar{u}_{n}\right|^{\alpha}+C_{10}\right)^{2}+\int_{0}^{T} \gamma(t) d t,
\end{aligned}
$$


which implies that $\left|\bar{u}_{n}\right|$ is bounded. Like in the proof of Lemma 3.1, we know that $\phi$ satisfies the P.S. condition.

Furthermore, we can prove Theorem 1.3 using the same way as in the proof of Theorem 1.2. Here, we omit it.

\section{Competing interests}

The authors declare that they have no competing interests.

\section{Authors' contributions}

All authors read and approved the final manuscript.

\section{Acknowledgements}

We express our gratitude to the referees for their valuable criticism of the manuscript and for helpful suggestions. Supported by the National Natural Science Foundation of China (11271371, 10971229).

Received: 24 April 2013 Accepted: 30 May 2013 Published: 25 June 2013

\section{References}

1. Mawhin, J, Willem, M: Critical Point Theory and Hamiltonian Systems. Springer, New York (1989)

2. Tang, CL: Periodic solutions of nonautonomous second order systems with sublinear nonlinearity. Proc. Am. Math. Soc. $126,3263-3270(1998)$

3. Ma, J, Tang, CL: Periodic solutions for some nonautonomous second-order systems. J. Math. Anal. Appl. 275, 482-494 (2002)

4. Jiang, Q, Tang, CL: Periodic and subharmonic solutions of a class of subquadratic second-order Hamiltonian systems. J. Math. Anal. Appl. 328, 380-389 (2007)

5. Luan, SX, Mao, AM: Periodic solutions of nonautonomous second order Hamiltonian systems. Acta Math. Sin. Engl. Ser. 21, 685-690 (2005)

6. Schechter, M: Periodic non-autonomous second-order dynamical systems. J. Differ. Equ. 223, 290-302 (2006)

7. Tang, CL: Periodic solutions of nonautonomous second order systems with $\gamma$-quasisubadditive potential. J. Math. Anal. Appl. 189, 671-675 (1995)

8. Tang, CL, Wu, XP: Periodic solutions for second order Hamiltonian systems with a change sign potential. J. Math. Anal. Appl. 292, 506-516 (2004)

9. Tao, ZL, Tang, CL: Periodic and subharmonic solutions of second-order Hamiltonian systems. J. Math. Anal. Appl. 293, 435-445 (2004)

10. Tao, ZL, Yan, SA, Wu, SL: Periodic solutions for a class of superquadratic Hamiltonian systems. J. Math. Anal. Appl. 331, 152-158 (2007)

11. Ye, YW, Tang, CL: Periodic solutions for some nonautonomous second order Hamiltonian systems. J. Math. Anal. Appl. 344, 462-471 (2008)

12. Yang, RG: Periodic solutions of some nonautonomous second order Hamiltonian systems. Nonlinear Anal. 69 , 2333-2338 (2008)

13. Tang, XH, Meng, Q: Solutions of a second-order Hamiltonian system with periodic boundary conditions. Nonlinear Anal., Real World Appl. 11, 3722-3733 (2010)

14. Zhou, JW, Li, YK: Existence of solutions for a class of second-order Hamiltonian systems with impulsive effects. Nonlinear Anal. 72, 1594-1603 (2010)

15. Nieto, JJ, O'Regan, D: Variational approach to impulsive differential equations. Nonlinear Anal., Real World Appl. 10 680-690 (2009)

16. Tian, Y, Ge, W: Applications of variational methods to boundary-value problem for impulsive differential equations. Proc. Edinb. Math. Soc. 51, 509-527 (2008) 